\title{
Au-Ag Alloy Nanoporous Nanotubes
}

\author{
Xiaohu Gu, Liqiang Xu, Fang Tian, and Yi Ding $(\varangle)$ \\ School of Chemistry and Chemical Engineering, Key Laboratory of Liquid Structure and Heredity of Materials, Ministry of Education, \\ Shandong University, Jinan 250100, China \\ Received: 13 February 2009 / Revised: 4 March 2009 / Accepted: 5 March 2009 \\ CTsinghua University Press and Springer-Verlag 2009. This article is published with open access at Springerlink.com
}

\begin{abstract}
Metallic nanostructures with hollow interiors or tailored porosity represent a special class of attractive materials with intriguing chemicophysical properties. This paper presents the fabrication of a new type of metallic nanoporous nanotube structure based on a facile and effective combination of nanocrystal growth and surface modification. By controlling the individual steps involved in this process, such as nanowire growth, surface modification, thermal diffusion, and dealloying, one-dimensional (1-D) metallic nanostructures can be prepared with tailored structural features and pre-designed functionalities. These tubular and porous nanostructures show distinct optical properties, such as tunable absorption in the near-infrared region, and enhanced capability for electrochemiluminescence signal amplification, which make them particularly desirable as novel 1-D nanocarriers for biomedical, drug delivery and sensing applications.
\end{abstract}

\section{KEYWORDS}

Nanoporous, nanotube, gold, optical properties, electrochemiluminescence

\section{Introduction}

Metallic nanostructures with hollow interiors or tailored porosity, such as nanotubes [1,2] and nanoporous membranes [3, 4], possess intriguing chemicophysical properties applicable in important fields including catalysis [5], biological labeling [6], surface enhanced Raman scattering (SERS) [7], thermal therapeutics $[8,9]$, and electrogenerated chemiluminescence (ECL) [10-12]. Therefore, great efforts have been devoted to the construction of such novel nanostructures with designed shape and geometry. Template-based synthesis is one of the most widely used methods for the fabrication of one-dimensional (1-D) nanostructures [13-15]. For example, gold nanotubes $[1,2]$ and nanoparticle nanotubes $[16,17]$ can be fabricated by templating porous alumina membranes using different surface modification techniques. Recently, based on a simple galvanic replacement reaction, $\mathrm{Xia}$ and co-workers have successfully synthesized a series of metallic hollow nanostructures with interesting properties, including nanoboxes, nanocages, and nanotubes [1820]. Herein, we report the fabrication of a new kind of metallic nanostructure called nanoporous nanotubes (NPNTs), in a process which allows flexible control of

Address correspondence to yding@sdu.edu.cn 
their individual structural parameters, such as tube dimension, pore/ligament size, and composition. Meanwhile, these novel nanostructures exhibit intense near-infrared (NIR) absorption and effective enhancement of ECL signal amplification, which suggest their great potential as novel 1-D nanocarriers for biomedical, drug delivery, and sensing applications.

Scheme 1 presents a schematic illustration of the major steps involved in the fabrication of $\mathrm{Au}-\mathrm{Ag}$ alloy NPNTs. It includes a three-step nanocrystal growth and structure-tailoring process. The first step is to synthesize Ag nanowires via a modified polyol process [18-20]. The next step is to carefully deposit a very thin layer of $\mathrm{Au}$ on the surface of the Ag nanowires. Upon controlled alloying of the $\mathrm{Au}$ surface layers with the Ag substrate under specific conditions, $\mathrm{Au} / \mathrm{Ag}$ surface alloys will form. While $\mathrm{Au}$ and $\mathrm{Ag}$ have distinct electrochemical properties, upon etching in nitric acid, Ag atoms from both the interior and the surface layer of the composite nanostructure can be controllably leached out, leaving behind metallic nanotubes with a welldefined porous wall structure.

\section{Experimental}

Materials. Silver nitrate $\left(\mathrm{AgNO}_{3}\right.$, A. R.), ethylene glycol (EG, A. R.), chloroauric acid $\left(\mathrm{HAuCl}_{4} \cdot 4 \mathrm{H}_{2} \mathrm{O}, \mathrm{A}\right.$. R.), ascorbic acid (AA, A. R.), cetyltrimethylammonium bromide (CTAB, A. R.), and concentrated nitric acid $\left(\mathrm{HNO}_{3}, 67 \%\right.$, A. R.) were purchased from Shanghai Sinopharm Chemical Reagent Co. Ltd. Polyvinylpyrrolidone (PVP, MW $\approx 55,000$ ) was purchased from Sigma-Aldrich. All chemicals were used as purchased without further purification. $\mathrm{HAuCl}_{4}$ aqueous solution $(1 \mathrm{mmol} / \mathrm{L})$ was prepared by dissolving $\mathrm{HAuCl}_{4} \cdot 4 \mathrm{H}_{2} \mathrm{O}$ in ultrapure water $(18.23 \mathrm{M} \Omega)$.

Synthesis of Ag nanowires. A modified polyol process was used to synthesize Ag nanowires. In a typical synthesis process, $5 \mathrm{~mL}$ of ethylene glycol (EG) was first placed in a three-neck flask, and heated in an oil bath at $160{ }^{\circ} \mathrm{C}$ for 10 min under magnetic stirring. Then, $3 \mathrm{~mL}$ of an ethylene glycol solution of $\mathrm{AgNO}_{3}(0.25 \mathrm{~mol} / \mathrm{L})$ and $3 \mathrm{~mL}$ of an ethylene glycol solution of PVP $(0.375 \mathrm{~mol} / \mathrm{L})$ were simultaneously

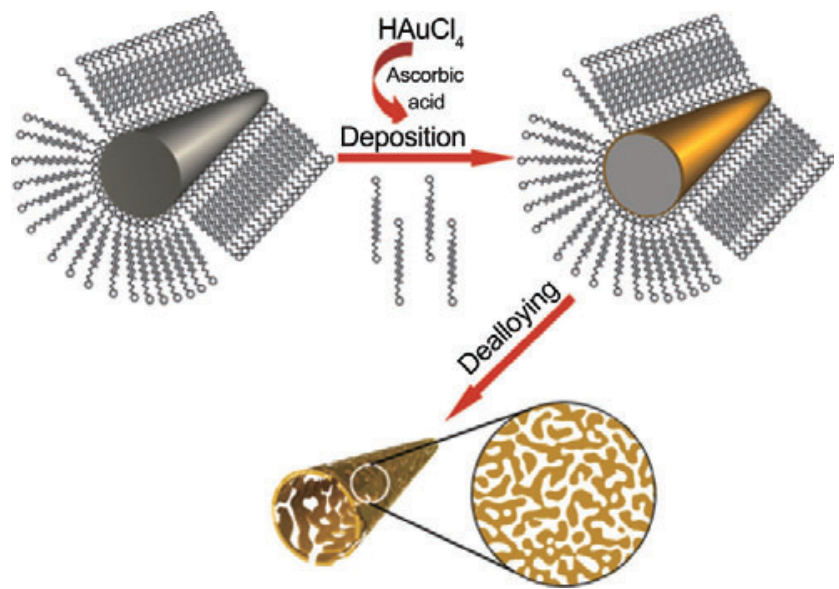

Scheme 1 Schematic illustration of the synthesis process of $\mathrm{Au}-\mathrm{Ag}$ alloy NPNTs

added through a two-channel syringe pump at a rate of $0.375 \mathrm{~mL} / \mathrm{min}$ to the solution under magnetic stirring. The solution was kept in the oil bath for another $30 \mathrm{~min}$. The final products (Ag nanowires) were collected by centrifugation, and then thoroughly washed with ethanol and ultrapure water to remove EG and PVP, and finally re-dispersed in ultrapure water for further use.

Fabrication of $\mathbf{A u}-\mathbf{A g}$ composite nanowires and NPNTs. In a typical synthesis, a fixed amount (0.02 $\mathrm{mol} / \mathrm{L}, 1 \mathrm{~mL}$ ) of $\mathrm{Ag}$ nanowires was dispersed in 17 $\mathrm{mL}$ of water containing $12 \mathrm{mmol} / \mathrm{L}$ of $\mathrm{CTAB}$ and 3 $\mathrm{mmol} / \mathrm{L}$ ascorbic acid in a $25 \mathrm{~mL}$ vial under magnetic stirring and then heated in a water bath at $40{ }^{\circ} \mathrm{C}$ for $10 \mathrm{~min}$. Then, $2 \mathrm{~mL}$ of $1 \mathrm{mmol} / \mathrm{L} \mathrm{HAuCl}_{4}$ solution was added dropwise to the vial. The solution was heated for another 25 min until the solution color became stable. The samples were centrifuged and washed with ultrapure water to remove excess $\mathrm{Cl}^{-}$, $\mathrm{CTAB}$, and ascorbic acid. Dealloying was carried out by re-dispersing the samples in $5 \mathrm{~mL}$ of ultrapure water, which was then mixed with a specific amount of concentrated nitric acid. After dealloying for 15 min, the samples were centrifuged and washed with ultrapure water and $\mathrm{NH}_{3} \cdot \mathrm{H}_{2} \mathrm{O}$ to remove excess nitric acid and trace amounts of $\mathrm{AgCl}$ before characterization.

ECL characterization. An Au disk electrode (4 $\mathrm{mm}$ in diameter) was polished carefully with $0.05-\mu \mathrm{m} \quad \alpha-\mathrm{Al}_{2} \mathrm{O}_{3}$ powder on fine abrasive paper and washed ultrasonically with ultrapure water. 
Before modification, the bare electrode was scanned in $0.5 \mathrm{~mol} / \mathrm{L} \mathrm{H}_{2} \mathrm{SO}_{4}$ between -0.65 and $+1.1 \mathrm{~V}$ vs a mercury-mercurous sulfate electrode (MSE), until a reproducible cyclic voltammogram (CV) was obtained. After cleaning, the electrode was first treated in a $0.02 \mathrm{~mol} / \mathrm{L}$ aqueous solution of cysteamine for $20 \mathrm{~h}$ in darkness at room temperature. For surface modification, the purified NPNTs were first added into an ethanol solution of $0.1 \mathrm{~mol} / \mathrm{L}$ thioglycolic acid in darkness at room temperature and left for 20 $h$, then centrifuged and re-dispersed in boric acid buffer solution $(\mathrm{pH}=7.4)$. The hybridization was performed by dipping the cleaned electrode into the as-prepared NPNTs solution at room temperature for $5 \mathrm{~h}$. After rinsing with ultrapure water, the modified electrode was immersed into $0.1 \mathrm{~mol} / \mathrm{L}$ phosphate buffer solution (PBS) $(\mathrm{pH}=7.4)$ containing $0.1 \mathrm{~mol} / \mathrm{L}$ $\mathrm{KCl}, 0.1 \mathrm{~mol} / \mathrm{L}$ tripropylamine (TPA), and $10 \mu \mathrm{mol} / \mathrm{L}$ $\mathrm{Ru}(\mathrm{bpy})_{3}^{2+}$ and scanned in the potential range from 0.2 to $1.25 \mathrm{~V}$ (vs Ag/ $\mathrm{AgCl}$ ) to record ECL signals.

Instrumentation. Scanning electron microscopy (SEM) and transmission electron microscopy (TEM) samples were prepared by placing a drop of the final products (dispersed in water) on a copper foil and carbon-coated copper grid, respectively, and dried under ambient conditions. SEM images were taken using a JEOL JSM-6700F field-emission scanning electron microscope operated at an accelerating voltage of $10 \mathrm{kV}$. TEM images and selected-area electron diffraction (SAED) were taken using a JEOL JEM-2100 high-resolution transmission electron microscopic (HRTEM) operated at an accelerating voltage of $200 \mathrm{kV}$. Composition measurements were conducted with an Oxford INCA X-sight Energy Dispersive X-ray Spectrometer (EDS) attached to the same microscope. The UV-visNIR spectra were recorded using a Hitachi U-4100 UV-vis-NIR spectrophotometer. A model MPI-E Electrochemiluminescence Analyzer Systems (Xi'an Remax Analytical Instrument Co. Ltd., Xi'an, China) was used to record the ECL signal. The voltage of the photomultiplier tube was set at $600 \mathrm{~V}$.

\section{Results and discussion}

Figure 1(a) shows an SEM image of the sacrificial Ag nanoparticle templates made by a modified polyol process [20], which are characterized by a highly monodisperse nanowire structure with diameter around $80 \mathrm{~nm}$. Adding $\mathrm{HAuCl}_{4}$ into a mixture of ascorbic acid and $\mathrm{Ag}$ nanowires gives facile reduction and deposition of $\mathrm{Au}$ atoms onto Ag nanowire surfaces [21]. Since the deposited Au overlayers are very thin, we found that even at a reduction temperature of $40{ }^{\circ} \mathrm{C}$, these surface $\mathrm{Au}$ atoms could spontaneously interdiffuse with the underlying $\mathrm{Ag}$ atoms to form $\mathrm{Au} / \mathrm{Ag}$ surface alloys [22]. This was proved not only by surface compositional analysis with XPS, but also by the fact that dealloying this nanocomposite in nitric acid generated metallic nanotubes with very characteristic nanoporous gold (NPG)-like [3,4] tube-wall structures. Figures 1(b)1(d) show typical SEM and TEM images of such an NPNT structure, which was made by depositing 2 $\mathrm{mL} \mathrm{HAuCl}_{4}(1 \mathrm{mmol} / \mathrm{L})$ onto $\mathrm{Ag}$ nanowires followed by etching in conc. $\mathrm{HNO}_{3}(3 \mathrm{~mL})$ for $15 \mathrm{~min}$. While these nanotubes retained very well the original 1-D structure and dimension of the Ag nanowire precursors, at higher magnification one can clearly

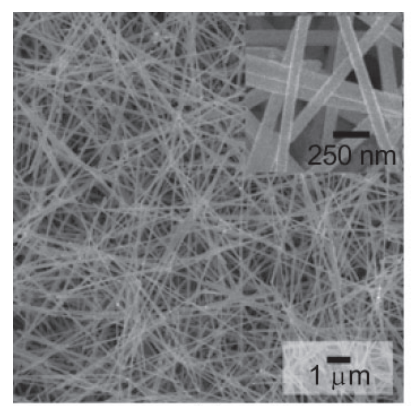

(a)

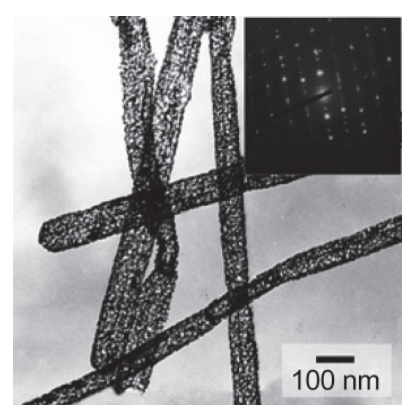

(c)

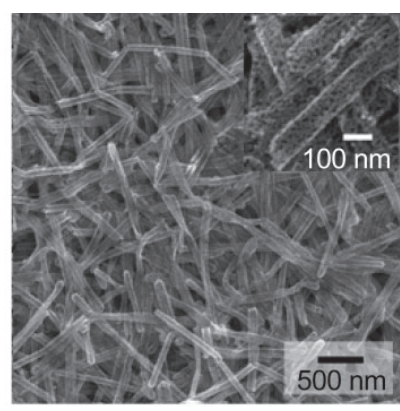

(b)

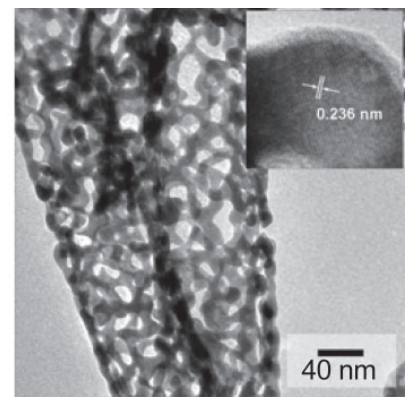

(d)
Figure 1 SEM and TEM images of Ag nanowire precursors (a) and Au-Ag alloy NPNTs ((b)-(d)). The insets are the corresponding higher magnification images, SAED pattern, and HRTEM image 
see that the tube walls are composed of interweaved ligaments and pores. Figure 1(d) shows an expanded view of two NPNTs, in which one can distinguish an $80 \mathrm{~nm}$ inner diameter nanotube with a single layer porous wall structure. The average ligament and pore sizes were determined to be 8 and $16 \mathrm{~nm}$, respectively. Similar to the porous gold structure made by dealloying bulk Au/Ag alloys [3, 4], these serpentine ligaments are not made of nanoparticles, but rather they adopt a locally single crystalline structure with continuous lattices spanning from one ligament to the other (Fig. S-1 in the Electronic Supplementary Material (ESM)). This feature was confirmed by electron diffraction and HRTEM. The clearly resolved lattice fringes with a period of $0.236 \mathrm{~nm}$ shown in the inset of Fig. 1(d) are typical for the $\{111\} d$-spacing of face centered cubic (fcc) structured $\mathrm{Au}$ (or Ag). The inset in Fig. 1(c) gives the electron diffraction pattern taken from an individual nanotube, which consists of at least two sets of diffraction spots with the major one corresponding to the [110] zone axis. modification with a small amount of $\mathrm{Au}(2 \mathrm{~mL}$ $\mathrm{HAuCl}_{4}$ ) led to an obvious red shift of the peak to around $590 \mathrm{~nm}$. Upon dealloying, the spectrum showed a further red shift to the NIR region. This shift was especially pronounced for the sample etched with $3 \mathrm{~mL}$ conc. $\mathrm{HNO}_{3}$, which had its main absorption peak well beyond $1200 \mathrm{~nm}$. Their intense NIR absorption suggests that these NPNTs may be effective as photo-thermal converters in biomedical applications such as drug release and cancer therapy $[8,9]$. Figures 2(b)-2(e) show typical TEM images for some of the samples. Sample II was decorated with approximately $2 \mathrm{~nm}$ Au. While core/shell structured composite $\mathrm{Ag} / \mathrm{Au}$ nanowires may have been expected, we actually found that certain portions of the nanowires already showed some degree of hollowness (marked by arrows in Fig. 2(b)). The reason can be ascribed to the local galvanic replacement between $\mathrm{HAuCl}_{4}$ and $\mathrm{Ag}$ nanowire templates, which has been demonstrated by Xia, albeit at higher temperature $\left(\sim 100{ }^{\circ} \mathrm{C}\right)$ [18-20].

The remaining spots can be ascribed to the existence of structural defects such as twin boundaries. Considering that Ag nanowires obtained from the polyol process usually adopt a twinned structure, the well-defined diffraction symmetry provides further evidence for an epitaxial relationship between the deposited Au and Ag substrate. Compositional analysis with energy dispersive spectroscopy (EDS, Fig. S-2 in the ESM) showed that this sample is in alloy form with a composition of $\mathrm{Au}_{65} \mathrm{Ag}_{35}$ (at\%).

The formation and structure evolution of the alloy NPNTs can also be monitored by spectroscopic methods, because $\mathrm{Au}$ and $\mathrm{Ag}$ nanostructures have very intense surface plasmon resonance peaks, which depend strongly on the structural parameters of the material $[23,24]$. Figure 2(a) shows a group of UV-vis-NIR absorption spectra of samples at different fabrication stages. While the Ag nanowires showed a characteristic absorption at $392 \mathrm{~nm}$ with a small shoulder peak at $385 \mathrm{~nm}$ due to their structural anisotropy [25], surface

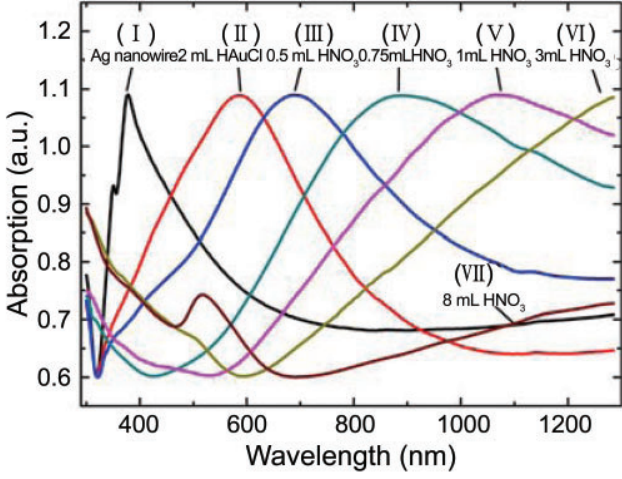

(a)

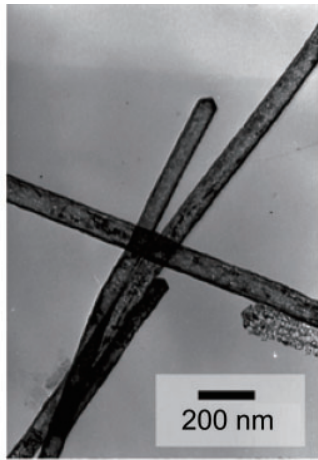

(c)

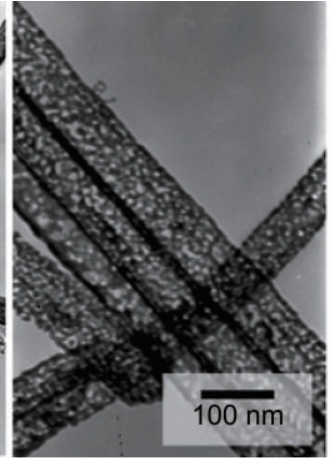

(d)

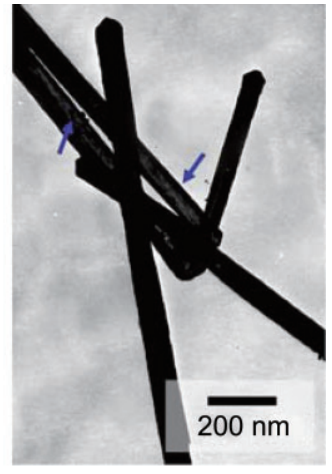

(b)

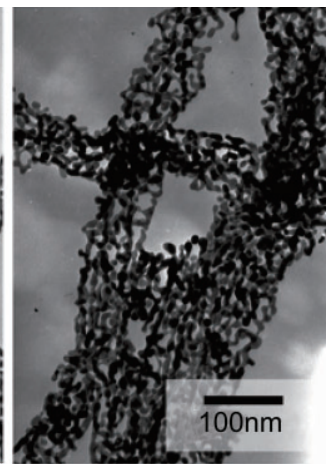

(e)
Figure 2 UV-vis-NIR absorption spectra (a) and TEM images ((b)-(e)) of samples I -VII at different fabrication stages. Images ((b)-(e)) correspond to samples II , V , VI, and VII, respectively 
However, it should be emphasized that in our experiment, most of the $\mathrm{Au}$ atoms are deposited through the redox reaction between $\mathrm{HAuCl}_{4}$ and the added reducing agent, ascorbic acid (see ESM). Control experiments showed that when gold was solely produced via the galvanic replacement between $\mathrm{HAuCl}_{4}$ and $\mathrm{Ag}$ nanowires (i.e., in the absence of ascorbic acid), the system was unstable for the following two reasons: (i) significant amounts of $\mathrm{AgCl}$ precipitate were formed, which influenced further reaction (Fig. S-3 in the ESM); and (ii) the 1-D nanostructures disintegrated into gold nanoparticles when the volume of $\mathrm{HAuCl}_{4}$ was increased to more than $4 \mathrm{~mL}$ (Fig. S-4 in the ESM). In contrast, in our system, even when the amount of $\mathrm{HAuCl}_{4}$ was increased to more than $16 \mathrm{~mL}$, the shape of the $\mathrm{Ag}$ nanowire templates was still well-preserved, and accordingly, there was a sustainable increase in nanowire diameter (Fig. S-5). After etching with $1 \mathrm{~mL}$ conc. $\mathrm{HNO}_{3}$, almost all the particles showed nanotube morphology although the porous wall structure was not fully developed (Fig. 2(c)). The best structure was observed after etching with $3 \mathrm{~mL} \mathrm{HNO}_{3}$ (Fig. 2(d)). It is interesting to note that further increasing the amount of $\mathrm{HNO}_{3}$ led to a significant coarsening of the porous structure, and eventual collapse of the NPNTs to chains of irregular nanoparticles (Fig. 2(e), $8 \mathrm{~mL}$ conc. $\mathrm{HNO}_{3}$ ). This structure change was also accompanied by a shift of its absorption back to the visible region around $520 \mathrm{~nm}$ (brown curve in Fig. 2(a), Sample VII), characteristic of gold nanoparticles, although there was still some absorption in the NIR region.

The structure evolution from Ag NWs to $\mathrm{Au}-\mathrm{Ag}$ alloy NPNTs was further investigated by changing the amount of the deposited gold. Interestingly, even when the amount of $\mathrm{HAuCl}_{4}$ was reduced to 0.5 $\mathrm{mL}$ (i.e., the nominal thickness of the deposited $\mathrm{Au}$ layer was about $1 \mathrm{~nm}$, assuming 100\% deposition efficiency), upon dealloying we still obtained $\mathrm{Au}$ -Ag alloy NPNTs with a continuous porous wall structure. Very surprisingly, however, we found that these alloy NPNTs exhibited rather different morphology. Figures 3(a) and 3(b) show typical TEM images of these NPNTs which have very thin tube walls. Compared with NPNTs with thicker walls (such as those shown in Fig. 2, deposited with 2 $\mathrm{mL} \mathrm{HAuCl}_{4}$ ), these NPNTs no longer possessed a robust and straight nanotube morphology. On the contrary, a highly curved rope-like nanostructure was observed, and in certain areas, they wound into a ring structure (indicated by an arrow in Fig. $3(b)$ ). A closer inspection of these nanoropes shows that the nanoporous tube structure was mostly preserved, although in certain areas the porous wall may have collapsed to form a deformed porous nanowire structure (Fig. 3(b)). Recently, there has been considerable research interest regarding the size-dependent mechanical properties of nanoporous metals [26]. Typically, very high yield strengths of nanoporous gold have been reported. The majority of these reports involve samples with ligament sizes of the order of several tens of nanometers in all three dimensions [26]. This is in marked contrast to our samples where the single layer porous wall structure has a ligament size typically less then 6 $\mathrm{nm}$. Apparently, these NPNTs with very thin porous walls exhibit ductile behavior and the electrostatic interaction between clean metal surfaces may result

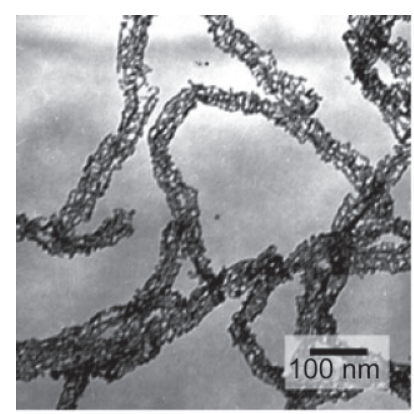

(a)

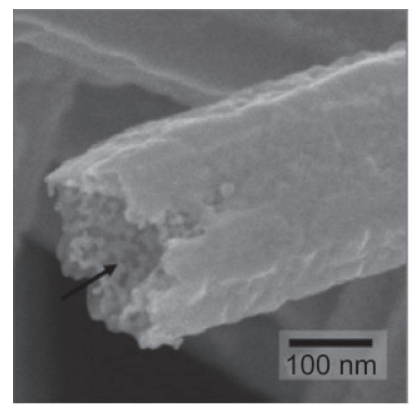

(c)

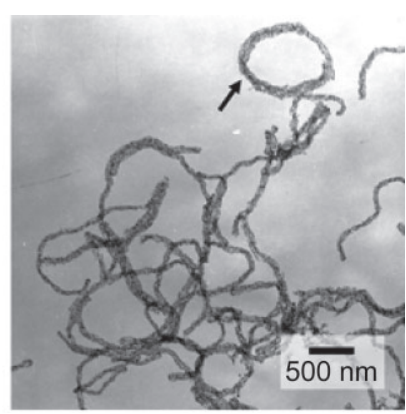

(b)

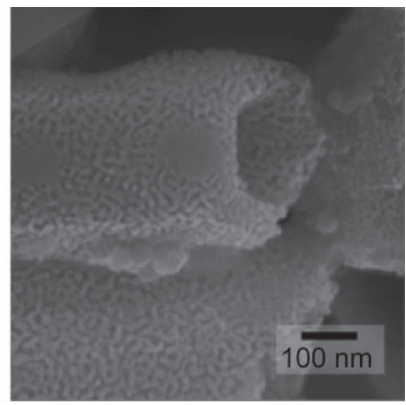

(d)
Figure 3 TEM micrographs of ((a), (b)) Au-Ag alloy NPNTs with very thin tube walls and ((c), (d)) Au-Ag alloy NPNTs with thick and gradient-structured porous walls 
in the aforementioned curved morphology. The actual mechanical properties of these NPNTs with their different wall structures are surely interesting topics that appear to warrant further investigations.

It should be noted that according to the previous literature, the galvanic replacement between $\mathrm{HAuCl}_{4}$ and sacrificial Ag nanoparticle templates can sometimes generate hollow nanostructures with holes of varying sizes in their shells [19]. The distinction of our approach is that the pores in the tube walls are generated via a highly controlled dealloying process. Not only the dimension of the Ag nanowire templates, but also the amount of surface Au deposited, and the alloying and dealloying conditions can be independently tailored; this allows a fine control of the structural parameters of the resulting NPNTs, including tube diameter, wall thickness, pore and ligament sizes, and composition. For example, Fig. 3(c) shows another kind of NPNT structure, which is characterized by a gradientstructured porous wall. With an inner diameter of $\sim 250 \mathrm{~nm}$, one can clearly see there are no apparent pores on the exterior surfaces of the nanotubes, while on the interior surfaces, we can see clear nanoporous structures just like NPG (marked by arrows). Briefly speaking, this is a tubular structure with roughened inner surfaces. In addition, by tailoring the thickness of the deposited $\mathrm{Au}$ surface layer and the alloying conditions between $\mathrm{Ag}$ and $\mathrm{Au}$, we can also generate NPNTs with truly three-dimensional porous wall structures as shown in Fig. 3(d).

ECL has become an important detection technique in bio-analytical chemistry in recent years [27]. ECL of luminol, lucigenin, tris $\left(2,2^{\prime}-\right.$ bipyridyl)ruthenium(II) $\left(\mathrm{Ru}(\mathrm{bpy})_{3}^{2+}\right)$, and their analogues on various electrode materials, such as glassy carbon (GC), gold, and platinum, has been well documented [10-12, 28]. Since Bard et al. opened the door to the exploration of ECL properties of various nanomaterials in 2002 [29], the preparation and applications of novel electrode materials have attracted considerable attention. Considering our NPNTs are rich in Au with very high surface area available for probe molecules, we were motivated to study their capability for the amplification of ECL signals for $\mathrm{Ru}(\mathrm{bpy})_{3}^{2+}$. The inset in Fig. 4 shows an
SEM image of the NPNTs tethered on the surface of an $\mathrm{Au}$ disk electrode, prepared by covalent bonding through cysteamine and thioglycolic acid. Although the coverage is quite low (less than $5 \%$ ), the modified electrode displays remarkably high ECL signals. Compared with a bare Au disk electrode, the NPNTsmodified electrode generated ECL intensity at least one order of magnitude larger at the oxidation potential of $\mathrm{Ru}(\mathrm{bpy})_{3}^{2+}(1.2 \mathrm{~V}$ vs $\mathrm{Ag} / \mathrm{AgCl})$. Compared with a cysteamine-covered $\mathrm{Au}$ disk electrode, the amplification value associated with the NPNTs represented an even more impressive increase of nearly 100 -fold. These preliminary investigations clearly demonstrate the effectiveness of NPNTs as highly efficient electrode materials for use in biosensing applications.

\section{Conclusions}

Novel metallic NPNTs have been fabricated based on an effective combination of nanocrystal growth and surface modification. By controlling the individual steps involved in this process, such as nanowire growth, surface modification, thermal diffusion, and dealloying process, a new class of 1-D metallic nanostructures can be prepared with tailored structural features and pre-designed functionalities. With porous surfaces and hollow interiors, these tubular nanostructures show very high surface areas as well as distinct optical properties, which make

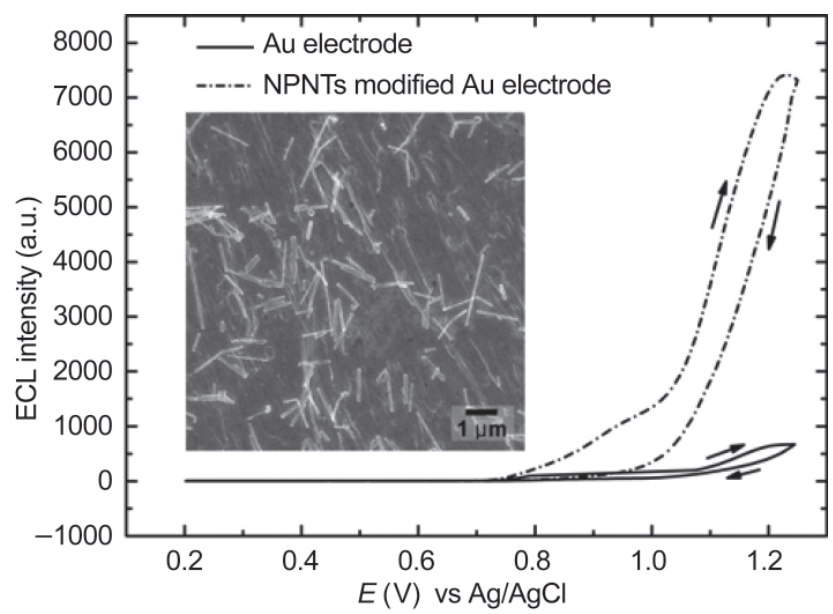

Figure 4 Electrochemiluminescence properties based on Au-Ag alloy NPNTs and a bare Au disk electrode. Inset is an SEM image of a Au disk electrode with NPNTs tethered by covalent bonding 
them particularly attractive for biomedical and sensing applications. Meanwhile, considering the wide variety of potential templating structures and modification materials, many new materials and structures can be fabricated in this way and should have novel functions, properties, and applications.

\section{Acknowledgements}

We thank Wei Liu, Qin Hao, Zhicheng Jü, and Prof. Wenrui Jin for their assistance in making Ag nanowires and in conducting the ECL experiments. This work was supported by the National 863 (2006AA03Z222) and 973 (2007CB936602) Program Projects of China, the Natural Science Foundation of Shandong Province (2007ZRB01117, 2006BS04018), and the Key Project of the Ministry of Education of China (108078). Y. D. is a Tai-Shan Scholar supported by the SEM-NCET, and SRF-ROCS Programs and the Shandong Natural Science Fund for Distinguished Young Scholars.

Electronic Supplementary Material: Five supplementary figures showing an HRTEM micrograph of the wall structure of a single NPNT, EDS analysis, and the effect of varying the composition of synthesis mixture on product morphology are available in the online version of this article at http://dx.doi.org/10.1007/s12274-009-9038-3 and are accessible free of charge.

\section{References}

[1] Martin, C. R. Nanomaterials-A membrane-based synthetic approach. Science 1994, 266, 1961-1966.

[2] Wirtz, M.; Parker, M.; Kobayashi, Y.; Martin, C. R. Template-synthesized nanotubes for chemical separations and analysis. Chem. Eur. J. 2002, 8, 3572-3578.

[3] Ding, Y.; Erlebacher, J. Nanoporous metals with controlled multimodal pore size distribution. J. Am. Chem. Soc. 2003, 125, 7772-7773.

[4] Ding, Y.; Kim, Y. J.; Erlebacher, J. Nanoporous gold leaf: "Ancient technology"/advanced material". Adv. Mater. 2004, 16, 1897-1900.

[5] Chen, Z.; Waje, M.; Li, W.; Yan, Y. Supportless Pt and PtPd nanotubes as electrocatalysts for oxygen-reduction reactions. Angew. Chem. Int. Ed. 2007, 46, 4060-4063.

[6] Chen, J.; Saeki, F.; Wiley, B. J.; Cang, H.; Cobb, M. J.; Li, Z. Y.; Au, L.; Zhang, H.; Kimmey, M. B.; Li, X. D.; Xia, Y. Gold nanocages: Bioconjugation and their potential use as optical imaging contrast agents. Nano Lett. 2005, 5, 473-477.

[7] Talley, C. E.; Jackson, J. B.; Oubre, C.; Grady, N. K.; Hollars, C. W.; Lane, S. M.; Huser, T. R.; Nordlander, P.; Halas, N. J. Surface-enhanced Raman scattering from individual Au nanoparticles and nanoparticle dimer substrates. Nano Lett. 2005, 5, 1569-1574.

[8] Hirsch, L. R.; Stafford, R. J.; Bankson, J. A.; Sershen, S. R.; Rivera, B.; Price, R. E.; Hazle, J. D.; Halas, N. J.; West, J. L. Nanoshell-mediated near-infrared thermal therapy of tumors under magnetic resonance guidance. Proc. Natl. Acad. Sci. USA 2003, 100, 13549-13554.

[9] Wu, G.; Mikhailovsky, A.; Khant, H. A.; Fu, C.; Chiu, W.; Zasadzinski, J. A. Remotely triggered liposome release by near-infrared light absorption via hollow gold nanoshells. J. Am. Chem. Soc. 2008, 130, 8175-8177.

[10] Gao, W.; Xia, X. H.; Xu, J. J.; Chen, H. Y. Threedimensionally ordered macroporous gold structure as an efficient matrix for solid-state electrochemiluminescence of Ru(bpy) ${ }_{3}^{2+} / \mathrm{TPA}$ system with high sensitivity. J. Phys. Chem. C 2007, 111, 12213-12219.

[11] Dong, Y. P.; Cui, H.; Wang, C. M. Electrogenerated chemiluminescence of luminol on a gold-nanorodmodified gold electrode. J. Phys. Chem. B 2006, 110, 18408-18414.

[12] Yin, X. B.; Qi, B.; Sun, X.; Yang, X.; Wang, E. 4-(dimethylamino)butyric acid labeling for electrochemiluminescence detection of biological substances by increasing sensitivity with gold nanoparticle amplification. Anal. Chem. 2005, 77, 3525-3530.

[13] Laocharoensuk, R.; Sattayasamitsathit, S.; Burdick, J.; Kanatharana, P.; Thavarungkul, P.; Wang, J. Shapetailored porous gold nanowires: From nano barbells to nano step-cones. ACS Nano 2007, 1, 403-408.

[14] Shin, T. Y.; Yoo, S. H.; Park, S. Gold nanotubes with a nanoporous wall: Their ultrathin platinum coating and superior electrocatalytic activity toward methanol oxidation. Chem. Mater. 2008, 20, 5682-5686.

[15] Ji, C.; Searson, P. C. Synthesis of nanoporous gold nanowires. J. Phys. Chem. B 2003, 107, 4494-4499.

[16] Lahav, M.; Sehayek, T.; Vaskevich, A.; Rubinstein, I. Nanoparticle nanotubes. Angew. Chem. Int. Ed. 2003, 
42, 5576-5579.

[17] Sehayek, T.; Lahav, M.; Popovitz-Biro, R.; Vaskevich, A.; Rubinstein, I. Template synthesis of nanotubes by roomtemperature coalescence of metal nanoparticles. Chem. Mater. 2005, 17, 3743-3748.

[18] Sun, Y.; Xia, Y. Multiple-walled nanotubes made of metals. Adv. Mater. 2004, 16, 264-268.

[19] Sun, Y.; Xia, Y. Mechanistic study on the replacement reaction between silver nanostructures and chloroauric acid in aqueous medium. J. Am. Chem. Soc. 2004, 126, 3892-3901.

[20] Sun, Y.; Xia, Y. Shape-controlled synthesis of gold and silver nanoparticles. Science 2002, 298, 2176-2179.

[21] Murphy, C. J.; Sau, T. K.; Gole, A.; Orendorff, C. J. Surfactant-directed synthesis and optical properties of one-dimensional plasmonic metallic nanostructures. MRS Bull. 2005, 30, 349-355.

[22] Shibata, T.; Bunker, B. A.; Zhang, Z.; Meisel, D.; Vardeman II, C. F.; Gezelter, J. D. Size-dependent spontaneous alloying of $\mathrm{Au}-\mathrm{Ag}$ nanoparticles. J. Am. Chem. Soc. 2002, 124, 11989-11996.

[23] Kim, F.; Connor, S.; Song, H.; Kuykendall, T.; Yang, P.
Platonic gold nanocrystals. Angew. Chem. Int. Ed. 2004, 43, 3673-3677.

[24] Seo, D.; Park, J. C.; Song, H. Polyhedral gold nanocrystals with $O_{\mathrm{h}}$ symmetry: From octahedra to cubes. J. Am. Chem. Soc. 2006, 128, 14863-14870.

[25] Sosa, I. O.; Noguez, C.; Barrera, R. G. Optical properties of metal nanoparticles with arbitrary shapes. J. Phys. Chem. B 2003, 107, 6269-6275.

[26] Volkert, C. A.; Lilleodden, E. T.; Kramer, D.; Weissmüller, J. Approaching the theoretical strength in nanoporous Au. Appl. Phys. Lett. 2006, 89, 061920.

[27] Fähnrich, K. A.; Pravda, M.; Guilbault, G. G. Recent applications of electrogenerated chemiluminescence in chemical analysis. Talanta 2001, 54, 531-559.

[28] Xu, X. H.; Bard, A. J. Electrogenerated chemiluminescence. 55. Emission from adsorbed Ru(bpy) ${ }_{3}^{2+}$ on graphite, platinum, and gold. Langmuir 1994, 10, 2409 2414.

[29] Ding, Z.; Quinn, B. M.; Haram, S. K.; Pell, L. E.; Korgel, B. A.; Bard, A. J. Electrochemistry and electrogenerated chemiluminescence from silicon nanocrystal quantum dots. Science 2002, 296, 1293-1297. 